SCIREA Journal of Traffic and Transportation

http://www.scirea.org/journal/TTE

March 9, 2021

Volume 4, Issue 1, February 2021

SCIREA

\title{
Initiatives of Open Science in Greece and the Transport Sector
}

\author{
Afroditi Anagnostopoulou ${ }^{*}$, Evangelos Spyrou ${ }^{2}$, Konstantinos Skoufas ${ }^{2}$ and Dimitrios \\ Mitrakos $^{2}$ \\ ${ }^{1}$ Centre for Research and Technology Hellas, Hellenic Institute of Transport *1 Kasimati Str., \\ 18531, Piraeus, Greece \\ ${ }^{2}$ Aristotle University of Thessaloniki, School of Electrical and Computer Engineering, \\ Greece \\ Email: a.anagnostopoulou@certh.gr (Afroditi Anagnostopoulou)
}

\begin{abstract}
This report presents Open Science initiatives in Greece and proposes modern services with respect to different challenges that stakeholders in transport may face. The focus is given on how Greece responded to the modern movement of openness promoted by the European Commission for increasing transparency, integrity and reproducibility of data, information and results. Different initiatives of Open Science are presented covering infrastructures, portals, data and other services. Finally, a modern system is proposed for the transport sector in an attempt to exploit previous research work and speed up the path from research to innovation and promote stakeholders' engagement.
\end{abstract}

Keywords: open data, infrastructures, modern services. 


\section{Introduction}

Open Science has been promoted in recent years by the European Commission (EC) [1] and initiatives on openness, transparency and democracy in knowledge dissemination and research have been intensified. As an integral part of the Digital Single Market and the European Research Area, Open Science is found in the EU Member States' research infrastructure roadmaps and proposed as an action of development locally and at a wider European level. In Greece, OS is at an early stage and several discussions initiated on the creation of a national "openness" framework that covers the needs of the entire life cycle of scientific research. Even though law 4310/2014 supports Open Access to publicly funded research, Greece has not implemented a national policy yet and recently, a Working Group has been established to support General Secretariat of Research and Technology [2] of the Ministry of Education and Religious Affairs [3] in order to develop a national strategy on Open Science aligned with the European Research Area (ERA) roadmap and present engagement and involvement of potential stakeholders.

Following this trend, the Technical University of Crete and the International Hellenic University in Greece have currently adopted an Open Access policy for their institutions based on the 2012 COM on access to and preservation of scientific information. Proper infrastructure has been developed and human resources with specialised know-how and expertise are available to support the development of OS in national level. There are sufficient institutional repositories that could host the research and academic production of the country's Universities. Network services, cloud computing and tools have already been developed for data analysis and an extensive and integrated legislative framework has been defined for public open data, open access support initiatives and open educational resources at national level (e.g. HEAL-Link). Athena Research and Innovation Center supports the development and adoption of Open Access and Open Science policies in Greece and acts as the National Open Access Desk (NOAD) by engaging key national stakeholders with an ultimate goal of delivering a national Open Science concept. However, Open Science in transport is lagging behind and is mainly focused on public transport services.

\section{Main Infrastructure in Open Science in Greece}

\subsection{Initiatives of National Documentation Centre}


The National Documentation Centre constitutes the national infrastructure which collects, organizes and preserves Greek scientific, research and cultural output (content and data) making it available for dissemination nationally and internationally. It provides wide-ranging information on the various research and innovation sectors, support participation in national and European programs, promote innovative entrepreneurship and the exploitation of research findings, and produce statistics \& indicators for research [4], development and innovation in Greece. The National Documentation Centre developed the National Information System for Research and Technology [5] to provide advanced electronic services in the world of research and innovation, foster open access to knowledge and ensure access to users regardless of time and location as described bellow.

\subsubsection{National Search Portals}

The National Documentation Centre has participated in the OpenArchives.gr project which aims to develop the largest portal providing a single point of access to Greek scientific digital content of high quality. Content providers are 59 [6] academic and research institutions, archives, libraries and scientific societies from Greece and Cyprus.

- The OpenAccess.gr has been also developed and supported by the National Documentation Centre in order to provide information on international and national developments in the area of Open Access.

- The MedOANet.eu addresses the necessity for coordinated strategies and policies in Open Access to scientific information in Europe. It aims to enhance existing policies, strategies and structures for Open Access and contributes towards the implementation of new ones in six Mediterranean countries: Greece, Turkey, Italy, France, Spain, and Portugal. The National Documentation Centre is the representative partner of the Greek area collaborating with the other Mediterranean countries.

- The SearchCulture.gr has been developed by the National Documentation Centre in an attempt to ensure the sustainability and reusability of content produced by publicly funded digitization projects, to provide central access to digital cultural resources.

- The Geodata.gov.gr provides geospatial data and services for Greece serving as a national open data catalogue. It has been developed in the context of EU-funded project PublicaMundi and maintained by IMIS/Athena RC [7]. This is a spatial data infrastructure that enables Open Geospatial Consortium services, interactive maps and data APIs utilizing open data. 


\subsection{Research Data Management Infrastructures}

HELIX initiative is funded by the OPERATIONAL PROGRAMME 'COMPETITIVENESS AND ENTREPRENEURSHIP' Programme and aims to develop the national e-Infrastructure in support of data-intensive research, handling the data management, analysis, sharing, and reuse needs of Greek scientists, researchers and innovators in a cross-disciplinary, scalable, and low-cost manner. HELIX is a joint effort of Athena Research \& Innovation Center and GRNET (National Infrastructures for Research and Technology) [8]. HELIX is the only horizontal e-Infrastructure of the National Roadmap for Research Infrastructures and is powered exclusively by open source software. The system is deployed over the national Okeanos-Knossos [9] cloud computing infrastructure and exposes the national HPC infrastructure ERMIS [10]. All provided publications are harvested from OpenAIRE as well as national and institutional Open Access repositories.

SCHOLIX aims to establish a high-level interoperability framework for exchanging information about the links between scholarly literature and data. It is a technical solution to wholesale information aggregation and supported by several organizations including OpenAIRE. In Greece, SCHOLIX will assist in the enhancement of repository metadata activities and through OpenAIRE, it will guide proper repository management and monitoring of data usage through the repository table service.

- The E $\Lambda / \Lambda \mathrm{AK}$ (Free Software / Open Source Software) [11] has been founded in 2008 and it is supported by 31 Universities, Research Centers and public benefit organizations. It aims to contribute to openness and in particular to the promotion and development of Open Standards, Free Software, Open Content, Open Data and Open Architecture Technologies in the field of education, public sector, business and social economy in Greece as well as to become a knowledge center and a platform for open technologies.

- NI4OS [12] is funded by the European Commission under the Horizon 2020 program and aims to be a core contributor to the European Open Science Cloud (EOSC) service portfolio, and aims to ensure inclusiveness on the European level for enabling global Open Science. It provides a training platform 18 about Open Science supporting the regional ICT and science sector by preventing brain drain. Athena Research \& Innovation Center and GRNET participate from Greece to this EU-funded initiative with the aim to empower the regional scientific and research community. 
- ELIXIR-GR [13] initiative brings together Research Infrastructures of the Greek National Roadmap19 including GRNET, Athena Research \& Innovation Center and Centre for Research and Technology Hellas. It is the Greek National Node of the ESFRI European RI ELIXIR which focuses on the construction of a sustainable European infrastructure for biological information. ELIXIRGR is funded by the EU Structural and Investment Funds and aims to supports life-science research and offers a catalogue of tools, services and benchmarks, ensuring best practices as well as sustainability and interoperability with other biological and medical science infrastructures.

- The Greek RDA Node [14] managed by Athena Research \& Innovation Center and aims to interact with researchers and innovators in the local language, offering them a platform for exchange of information pertinent to the RDA and their activities and in strict compliance with RDA's guiding principles of Openness, Transparency, Consensus-based, Community driven, Harmonization and Non-profit. Figure 1 presents the different sectors participating in the Greek RDA and it is reasonable that Academia/Research constitute the majority of the members as RDA is an important source of knowledge on a variety of research data issues.
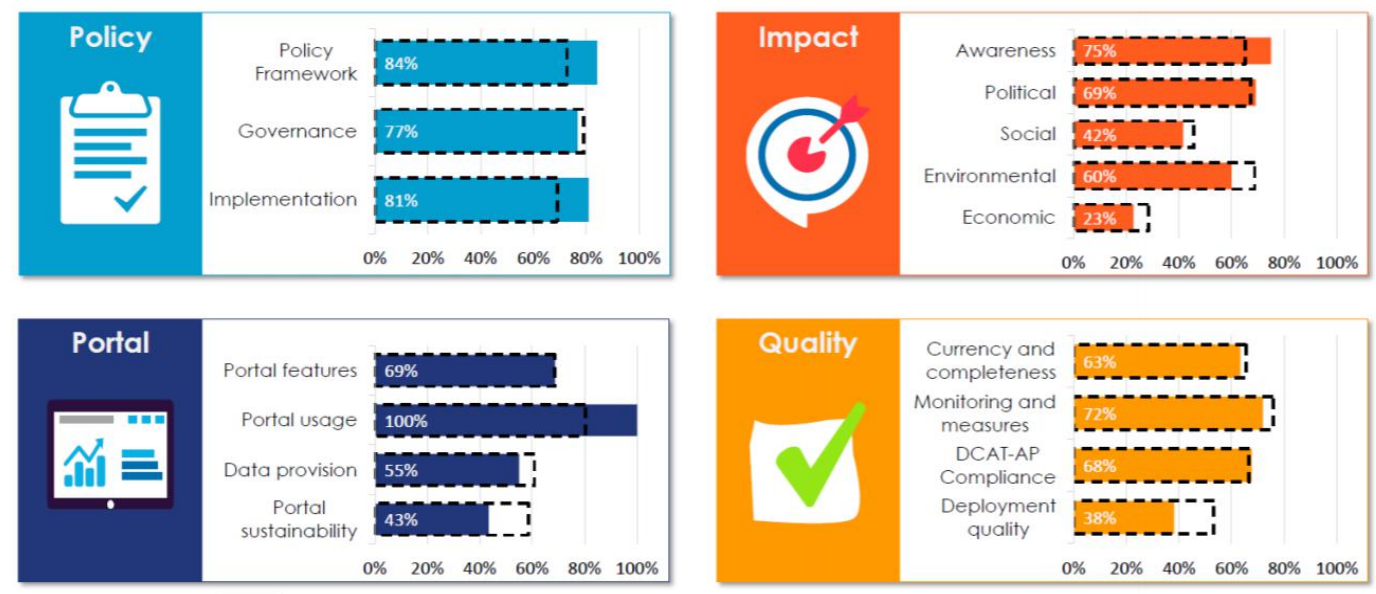

L-:-1 Eu28 Average $\times \%$ Greece

Figure 1: Performance of Greece.

Source: European Data Portal [15]

\subsection{OpenAIRE, the National Perspective}

The main focus of OpenAIRE [16] in Greece is to support stakeholders in their Open Science activities by transferring knowledge and experience in order to align national initiatives with EU goals. As such, OpenAIRE establishes strong collaborations with key national 
stakeholders as well as work with policymakers, funders and research performing organisations. OpenAIRE provides guidance, tools and policies through workshops and webinars enhancing EOSC initiative and formulating the national OS strategy and policy framework based on each country's needs and in line with EU directions.

\section{Open Science in Transport in Greece}

Open Science in Transport Research requires precisely defined data, adjusted incentives and necessary training to researchers in order to use OS tools. Data collection standards, metadata, infrastructure and functional requirements should also be defined in order to ensure an appropriate level of service [17]. In Greece, Open Science in Transport is mainly used for public transport services (i.e. OASA [18] provides real-time information for buses and trolleys in Athens) and OpenAIRE utilizing tools like NI4OS and RDA could provide proper training for enhancing their use. Moreover, European Open Science Cloud (EOSC) could provide services for disseminating knowledge and sharing research data. Transport researchers in Greece have understood the opportunity of EOSC that could provide not only a detailed service catalogue but also data protection and observation policies.

\subsection{Open Data and Modern Transport Services}

In transport sector, data could provide the basis for minimizing traffic congestion in modern cities. Data about traffic and unexpected events in the road network of a city could be provided from different stakeholders (i.e. services of Region and Municipality, Traffic Police, Ministries, citizens etc.) and by making them Open the development of a cooperative model for optimizing routing and navigation of vehicles could be achieved.

In an attempt to develop a technologically advanced vehicle routing system that is evolved dynamically, open data and real-time information are required in order to optimize navigation of vehicles in an urban region of a city. The main goal of this system is to effectively drive vehicles by incorporating the arrival of new road traffic data and/or the renewal of old data in real time.

More specifically, proper installations (i.e. optical light cameras and sensors infrared) at traffic lights are able to measure the passing vehicles and the system operates as follows (Figure 2): 
1. The traffic light devices receive the data from the vehicles, namely, their unique number to calculate the queue, their speed, their direction, and in conjunction with environmental data, they send it to the central server.

2. The vehicle devices send the GPS coordinates as well as other information from the On board Diagnostics (OBD-II) reader to the server.

3. The central server calculates the weights for the Backpressure algorithm to operate [18].

4. The central server sends the weights to the OSRM server, the OSRM server calculates the optimal path and sends it to the vehicle that requested it. In the case that the vehicle is stopped at a junction, the algorithm checks if the optimal path has changed and if yes, the new route is sent to the vehicle.

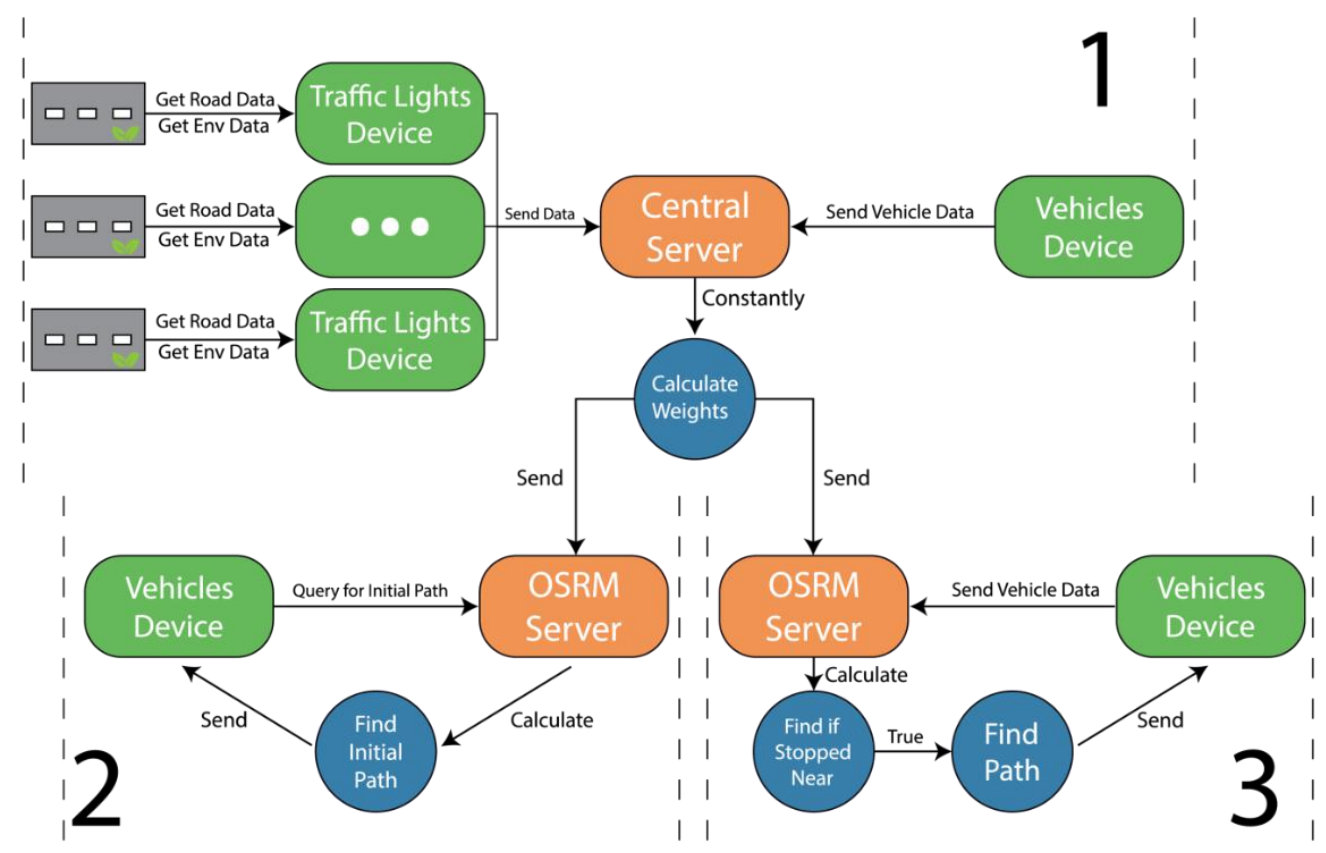

Figure 2: System operation.

A pilot activity of the proposed technologically advanced vehicle routing system was implemented in the city of Thessaloniki in the context of a co-financed research project by the European Regional Development Fund of the European Union and Greek national funds. Emerging technologies of Vehicle to Infrastructure (V2X), mobile communication networks as well as Internet of Things (IoT) were used allowing communication of server, traffic lights and vehicles. Dynamic distributed algorithms could be developed based on the backpressure approach [18] to ensure the cooperation of the system with the vehicles allowing automation 
that aims to achieve traffic minimization and optimal routing and navigation of different type of vehicles (i.e. corporate vehicles [19], electric vehicles [20]). A cloud infrastructure was also used to store the data (i.e. passing vehicles and their speed, queues in traffic lights, unexpected events) from the studied greater area of Thessaloniki city.

By providing proper infrastructure in order to cover the total road network of a city and by making these data open, interested stakeholders could easily access them for developing a technologically advanced vehicle routing system (figure 3) as the proposed. Moreover, additional services such as monitoring vehicles, minimizing corresponding environmental inefficiencies etc. that could provide a higher level of service for their transportations and better quality of life to residents of a city.

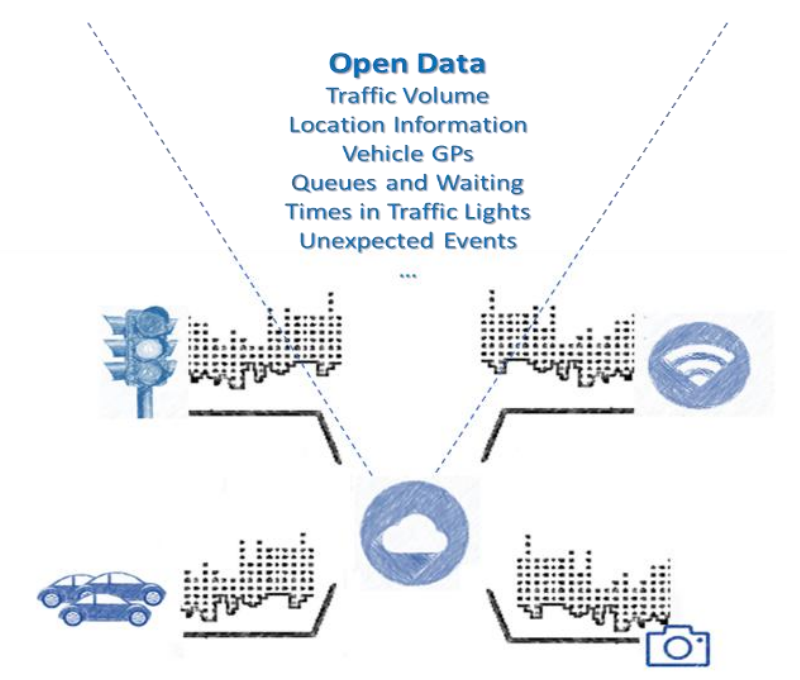

Figure 3: A technologically advanced vehicle routing system.

\section{Conclusions}

According to the analysis of Open Science initiatives in Greece and previous research in transport sector, this report presents how transport related data could be used to develop modern services by making them open. In an attempt to increase efficiency of transportation in urban areas, network services, cloud computing, data management infrastructures and other tools developed in Greece could be used and updated in order to provide transparent and understandable processes that will allow the development of an innovative transport system capable to cover stakeholders' needs and requirements. 


\section{Acknowledgements}

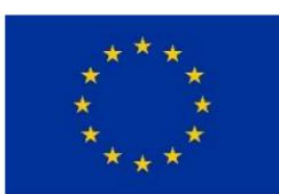

This project has received funding from the European Union's Horizon 2020 research and innovation programme under grant agreement No 824323. The results in this paper reflect only the authors' view. Neither the Innovation and Network Executive Agency nor the European Commission is responsible for any use that may be made of the information contained therein.

$\&$

This research has been co-financed by the European Regional Development Fund of the European Union and Greek national funds through the Operational Program Competitiveness, Entrepreneurship and Innovation, under the call RESEARCH - CREATE - INNOVATE (project code: T1EDK - 04012).

\section{References}

[1] A. Tsakalidis, K. Gkoumas, F. Pekar, M. Grosso, G. Haq and L. Marelli, "EU Transport Research \& Innovation Status Assessment Report 2017”, Publications Office of the European Union, 2018.

[2] General Secretariat of Research and Technology, http://www.gsrt.gr/central.aspx?sId=119I428I1089I323I488743.

[3] Ministry of Education and Religious Affairs, https://www.minedu.gov.gr/.

[4] MetricsEKT, Indicatos and statistics for Research, Development, Innovation, https://metrics.ekt.gr/en

[5] National Information System for Research and Technology, http://www.epset.gr/en.

[6] Academic and Research Institutions Content Providers, https://www.openarchives.gr/aggregator-openarchives/portal/institutions.

[7] Information Management Systems Institute http://www.imsi.athenarc.gr/.

[8] GRNET - National Infrastructures for Research and Technology, https://grnet.gr/en/.

[9] Okeanos-Knossos Cloud Computing Infrastructure, https://okeanosknossos.grnet.gr/home/.

[10] HPC Infrastructure ERMIS, https://hpc.grnet.gr/en/.

[11] E $\Lambda / \Lambda \mathrm{AK}$ (Free Software / Open Source Software), https://ellak.gr/.

[12] NI4OS, https://ni4os.eu/. 
[13] ELIXIR-GR, https://www.elixir-greece.org/

[14] RDA Node Greece, https://www.rd-alliance.org/groups/rda-greece.

[15] European Data Portal, https://www.europeandataportal.eu/en.

[16] OpenAIRE Greece, https://www.athenarc.gr/en/openaire-greece.

[17] G. Yannis, M. Böhm, J. R. Franklin, S. Jones, T. Kovacikova, K. Nowicka, K. Folla

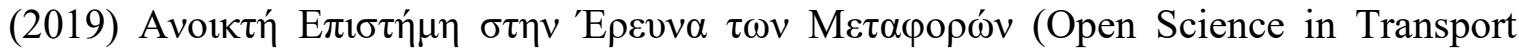
Research). 9th International Congress on Transportation Research, 24th-25th October, Athens, Greece.

[18] S. Moeller, A. Sridharan, B. Krishnamachari, O. Gnawali, (2010). Routing without routes: The backpressure collection protocol. In Proceedings of the 9th ACM/IEEE International Conference on Information Processing in Sensor Networks (pp. 279-290).

[19] A. Anagnostopoulou, E. Spyrou, D. Mitrakos, M. Boile, (2019). An advanced solution for efficient logistics management at both company and network level. Transportation Planning and Technology, 42(4), 324-338.

[20] E. D. Spyrou, D. Mitrakos, (2016). A backpressure framework applied to road traffic routing for electric vehicles. In Proceedings of the Sixth International Symposium on Business Modeling and Software Design (Vol. 1, pp. 235-240). 